\title{
Arterial Supply of Sciatic Nerve and Its Impact on Clinical Practice
}

\author{
EI Sayed Aly Mohamed Metwally ${ }^{1}$, Nancy Mohamed Aly El-Sekily ${ }^{1}$, \\ Naguib Abd El Karim Ramadan ${ }^{2}$ \\ ${ }^{1}$ Department of Anatomy and Embryology, Faculty of Medicine, Alexandria University, Alexandria, Egypt \\ ${ }^{2}$ General Surgery Department, Faculty of Medicine, Alexandria University, Alexandria, Egypt
}

Email address:

sayedmetwally2020@yahoo.com (E. S. A. M. Metwally), nancyelsekily@yahoo.com (N. M. A. El-Sekily), naguib@yahoo.com (N.A.E. Ramadan)

\section{To cite this article:}

El Sayed Aly Mohamed Metwally, Nancy Mohamed Aly El-Sekily, Naguib Abd El Karim Ramadan. Arterial Supply of Sciatic Nerve and Its Impact on Clinical Practice. International Journal of Clinical and Developmental Anatomy. Vol. 1, No. 3, 2015, pp. 79-84.

doi: $10.11648 /$ j.ijcda.20150103.14

\begin{abstract}
Sciatic nerve is the nerve of the posterior compartment of thigh; it is formed in the pelvis from the ventral rami of L4 to S3 spinal nerves. It leaves the pelvis via the greater sciatic foramen below piriformis and divides into common peroneal nerve and tibial nerve at the level of the upper angle of the popliteal fossa. The vasculature of peripheral nerves is adapted specifically to their structure and function. Arterial vessels that reach main nerve trunks originate from the adjacent main arteries or their muscular or cutaneous branches. Although variability in arterial supply of sciatic nerve was discussed by some authors it is still deficient. To minimize sciatic nerve ischemia and its concomitant problems, an understanding of the origin and course of the arteries supplying it in the gluteal region and the posterior compartment of thigh is important. The present study aimed to detect the different arteries supplying the sciatic nerve in the gluteal and posterior thigh regions and to study the importance of this in clinical practice. Three cases subjected to vascular surgery and twenty lower limbs of ten formalin preserved male cadavers were used. Higher division of sciatic nerve was observed in all specimens. In 6 specimens (30\%), arterial supply of sciatic nerve originated from the inferior gluteal artery, 6 specimens $(30 \%)$ from first, second and third perforating arteries, 5 specimens (25\%)from the lateral circumflex femoral artery and 3 specimens (15\%)from internal pudendal artery. Anastomosis between internal iliac artery through its internal pudendal branch and external iliac artery through perforating arteries was observed. It was concluded that there is a good anastomosis between internal iliac and profunda femoris artery on the same side but cross pelvic anastomosis is absent or deficient and in case of profunda femoris occlusion we should preserve internal pudendal artery and the reverse.
\end{abstract}

Keywords: Sciatic Nerve, Ischemia, Gluteal Artery, Pudendal Artery, Perforating Artery

\section{Introduction}

Sciatic Nerve (SN) is the nerve of the posterior compartment of thigh formed in the pelvis from the ventral rami of L4 to S3 spinal nerves. It leaves the pelvis via the greater sciatic foramen below piriformis and divides into common peroneal nerve $(\mathrm{CPN})$ and tibial nerve $(\mathrm{TN})$ at the level of the upper angle of the popliteal fossa (1). The sciatic nerve enters the gluteal region posterior to obturator internus and the underlying hip joint capsule. As it leaves the pelvis, the relationship of the sciatic nerve to piriformis is variable, some configurations of which may lead to compression of the nerve, particularly during total hip arthroplasty (2). Higher division of the sciatic nerve is the most common variation where the $\mathrm{TN}$ and $\mathrm{CPN}$ may leave the pelvis through different routes. Such variation may lead to compression of the nerve and lead to non-discogenic sciatica (1).

The vasculature of peripheral nerves is adapted specifically to their structure and function. Arterial vessels (vasa nervosa) which reach main nerve trunks originate from the adjacent main arteries or their muscular or cutaneous branches. The number, length, caliber, and origin of vasa nervosa, as well as their pattern on approach to nerve trunks vary not only between different nerves but between different parts of the same nerve (1). Mutual anastomosis of these arteries gives extraneural networks located in the epineurium. These arterial networks then give branches along the 
perineurium which anastomose and form intraneural arterial networks. Generally, the closer the vascular network to nerve fibers, the more subtle it becomes(3). Variability in arterial supply of sciatic nerve was discussed by some authors $(4,5,6,7,8,9)$. But it is still deficient. Sciatic nerve injury is a possible complication of hip replacement due to injury of the nerve itself, or its blood vessels $(10,11)$.

To minimize sciatic nerve ischemia and its concomitant problems, an understanding of the origin and course of the arteries supplying it in the gluteal region and the posterior compartment of thigh is important. Appropriate steps can be taken during surgical procedures, such as hip arthroplasty, to avoid direct or indirect damage to the artery itself or its parent vessel.

\section{Material \& Methods}

\subsection{Materials and Subjects}

The clinical part of the study included 3 case reports; case one was a 37 years old male patient, addict with infected femoral pseudoaneurysm at femoral bifurcation. Ligation of profunda femoris and internal iliac arteries simultaneously was done, with revascularization of the limb through extraanatomic iliosuperficial femoral bypass using reversed saphenous vein graft. Case two was a 63 years old male patient with chronic lower limb ischemia, short segment occlusion of proximal external iliac artery with chronic occlusion of profunda femoris artery during percutaneous transluminar angioplasty (PTA) and stenting of the external iliac lesion. internal iliac artery was accidently occluded by dissection flap. Case three was a 67 years old female patient with chronic lower limb ischemia with occlusion of internal iliac artery. Angiography and electrophysiological studies were done for all cases.

The anatomical part of the study included twenty lower limbs of ten formalin preserved male cadavers obtained from Anatomy Department, Faculty of Medicine, Alexandria University. The gluteal and posterior thigh regions were exposed and carefully dissected.

An initial incision was made from the posterior superior iliac spine along the iliac crest to the iliac tubercle, and then continued inferiorly as far as the distal third of the posterior thigh till the popliteal fossa. The resulting skin flap was reflected medially. Gluteus maximus was reflected to expose the sciatic nerve. The arterial supply of the sciatic nerve between its emergence from the greater sciatic foramen till its division was determined also the mean length of these arteries was measured using Vernier caliper.

\subsection{Statistical Analysis}

Results were expressed as means \pm standard deviation

$(\mathrm{M} \pm \mathrm{SD})$. Statistical analysis was performed using Statistical package for social science (SPSS) version 16.0. Data were analyzed using one-way analysis of variance (ANOVA) and significant difference was determined using post-Hoc Turkey's test for multiple comparisons at $(\mathrm{p}<0.05)$.

\section{Results}

\subsection{Clinical Results}

In case one, simultaneous ligation of profunda femoris and internal iliac arteries with revascularization of the limb through extraanatomic iliosuperficial femoral bypass using reversed saphenous vein graft resulted in paresis of the limb inspite of the felt distal pulsations. Also in case two, occlusion of profunda femoris artery and accidental interruption of internal iliac artery blood flow by dissection flap resulted in sciatic ischemia. While in case three, occlusion of internal iliac artery with patent external iliac, common femoral and profunda femoris with superficial femoral artery (SFA), accidental interruption of profunda femoris artery (during construction of femoropopliteal bypass) resulted in lower limb paresis and sciatic ischemia.

Sciatic neuropathy was confirmed by electrophysiological studies in all acses.

\subsection{Anatomical Results}

Beginning, course and termination of sciatic nerve:

In all the specimens the sciatic nerve emerged below piriformis muscle and covered by gluteus maximus. There is high division of sciatic nerve into common peroneal and tibial component; in 6 out of 20 specimens $(30 \%)$ it was in the gluteal region (Fig. 1), in 6 out of 20 specimens $(30 \%)$ it was in the upper third of thigh (Fig. 2a, b) and in 8 out of 20 specimens $(40 \%)$ it was in the middle of thigh (Fig. 3,4,5) (Table 1).

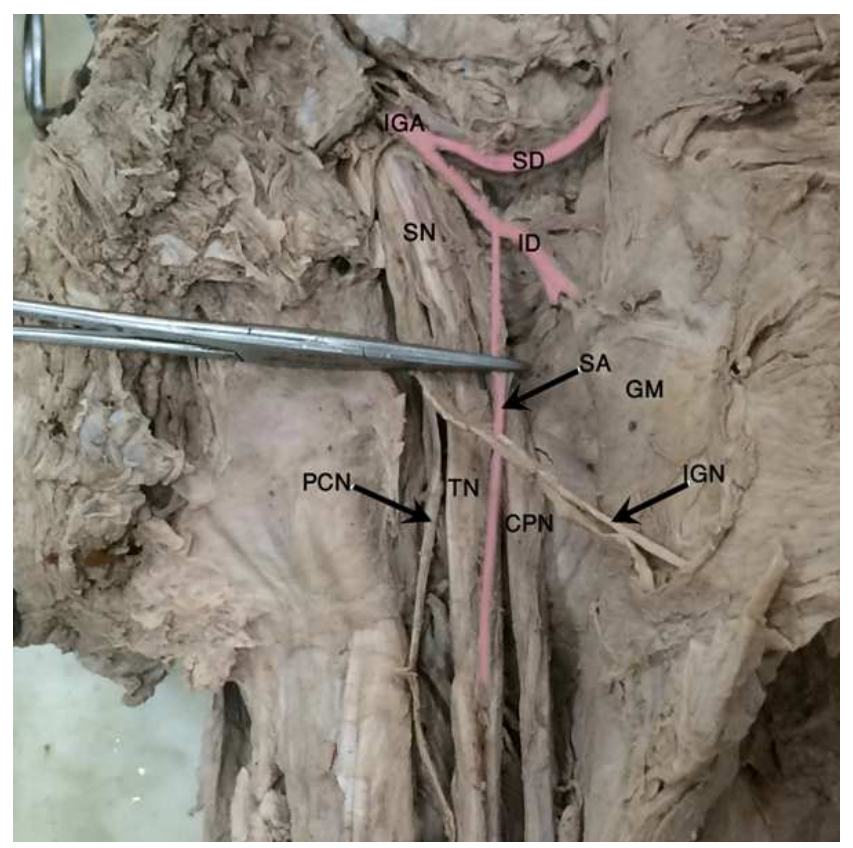

Fig. 1. A photograph of right lower limb with dissected gluteal region showing inferior gluteal artery (IGA) divided into superior division(SD) and inferior divison (ID) supplying gluteus maximus (GM).IGA gave sciatic $\operatorname{artery}(S A)$ supplying the sciatic nerve $(S N)$. The $(S A)$ continued into the tibial component (TN) of (SN).CPN: common peroneal nerve, PCN: posterior cutaneous nerve of thigh.Note the higher division of $S N$ in gluteal region. 
Table 1. Variability of the level of division of the sciatic nerve in 20 cadaveric specimens.

\begin{tabular}{ll}
\hline Level of division & Number of specimens \\
\hline Gluteal region & $6(30 \%)$ \\
Upper thigh & $6(30 \%)$ \\
Middle thigh & $8(40 \%)$ \\
Total & $20(100 \%)$ \\
\hline
\end{tabular}

Arterial supply of sciatic nerve:

The arterial supply of sciatic nerve was identified in all the specimens, their origin was variable. Six out of 20 specimens $(30 \%)$ it originated from the inferior gluteal artery (Fig. 1, $3), 6$ out of 20 specimens (30\%) from first, second and third perforating arteries (Fig.2a,b, 4), 5 out of 20 specimens $(25 \%)$ from the lateral circumflex femoral artery (Fig. 5) and 3 out of 20 specimens (15\%) from internal pudendal artery (Fig. 2b) (Table 2).

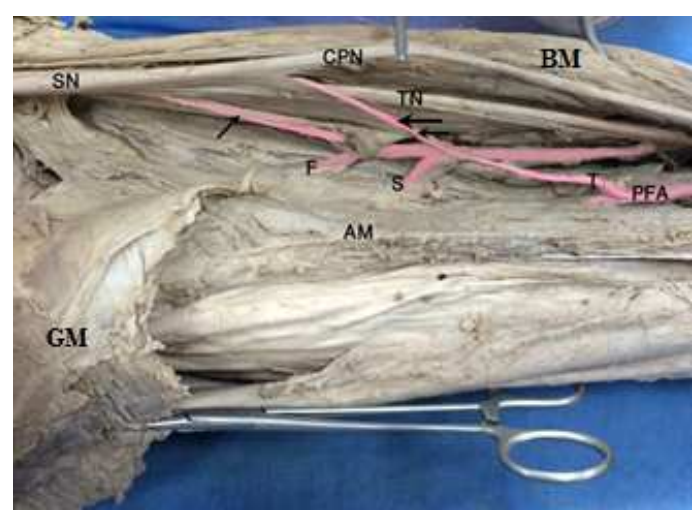

Fig. 2a. A photograph of left lower limb with dissected posterior thigh region showing the profunda femoris artery (PFA) giving 1st $(F)$, 2nd $(S)$ and $3 r d(T)$ perforators. The three perforators $(F, S, T)$ pierced the adductor magnus muscle (AM).The $(F)$ and $(S)$ formed an anastomotic channel (arrow) which supplied the sciatic nerve $(S N)$. The $(T)$ perforator gave a branch (double arrow) which supplied the (SN). BM: biceps femoris muscle, $C P N$ : common peroneal nerve, TN: tibial nerve, GM: gluteus maximus (reflected). Note the higher division of SN in upper third of thigh.

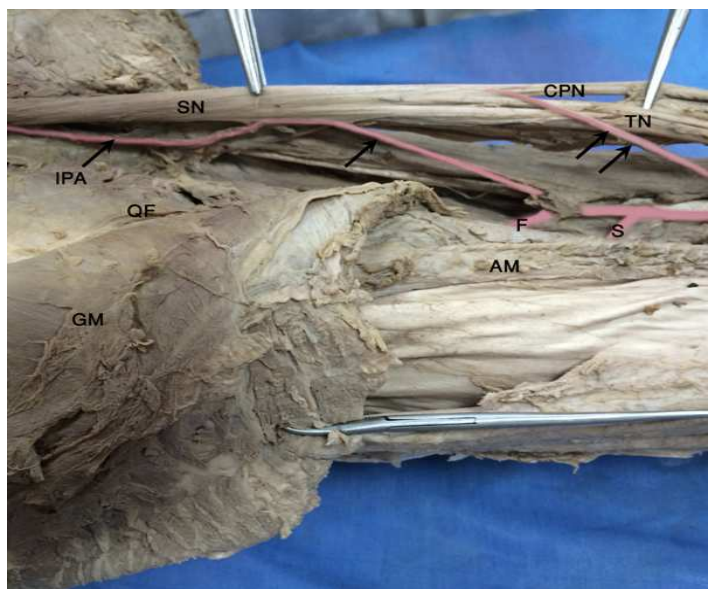

Fig. 2b. A photograph of the previous specimen (higher level) showing the internal pudendal artery (IPA) supplying the anterior aspect of sciatic nerve $(S N)$. 1st $(F)$, 2nd (S) perforators pierced the adductor magnus muscle $(A M)$.The $(F)$ and $(S)$ formed an anastomotic channel (arrow) which anastomosed with (IPA). Double arrow: branch of third perforator, CPN: common peroneal nerve, TN: tibial nerve, GM: gluteus maximus (reflected), QF: qudratus femoris.
Table 2. The source and total number of arteries supplying the sciatic nerve in 20 cadaveric specimens.

\begin{tabular}{ll}
\hline Artery & Number of specimens \\
\hline Inferior gluteal artery & $6(30 \%)$ \\
First, second and third perforating arteries & $6(30 \%)$ \\
Lateral circumflex femoral artery & $5(25 \%)$ \\
Internal pudendal artery & $3(15 \%)$ \\
\hline
\end{tabular}

The sciatic artery originated from inferior division of IGA supplied the posterior aspect of $\mathrm{SN}$ and continued with its tibial component (Fig. 1). Also the IGA gave 2 branches to supply the posterior aspect of SN (Fig. 3)

The 1st and 2nd perforating arteries form an anastomotic channel from which an artery ascends to supply the upper part of sciatic nerve (Fig. 2a).

The third perforating artery ascends upward to supply the terminal part of SN and proximal part of its tibial component (Fig. 2a).

IPA emerged posterior to quadratus femoris to supply the anterior aspect of $\mathrm{SN}$ and anastomosed with the anastomosing branch of the first and second perforating arteries (Fig.2b).

Length of the arteries supplying sciatic nerve:

The mean length of the branches from the inferior gluteal artery was $34.9 \pm 1 \mathrm{~mm}$, third perforating artery was $26.7 \pm$ $0.2 \mathrm{~mm}$, from the anastomotic channel of the first and second perforating arteries was $22.6 \pm 1 \mathrm{~mm}$, from internal pudendal artery was $20 \pm 1 \mathrm{~mm}$ and from lateral circumflex femoral artery was $17 \pm 1 \mathrm{~mm}$ (Table 3 ).

Table 3. The mean length of the arteries supplying the sciatic nerve in 20 cadaveric specimens.

\begin{tabular}{ll}
\hline Artery & Length in $\mathbf{~ m m}$ \\
\hline Branches from IGA & $34.9 \pm 1$ \\
Third perforating artery & $26.7 \pm .2$ \\
First and second perforating artery & $22.6 \pm 1$ \\
IPA & $20 \pm 1$ \\
Lateral circumflex femoral artery & $17 \pm 1$ \\
\hline
\end{tabular}

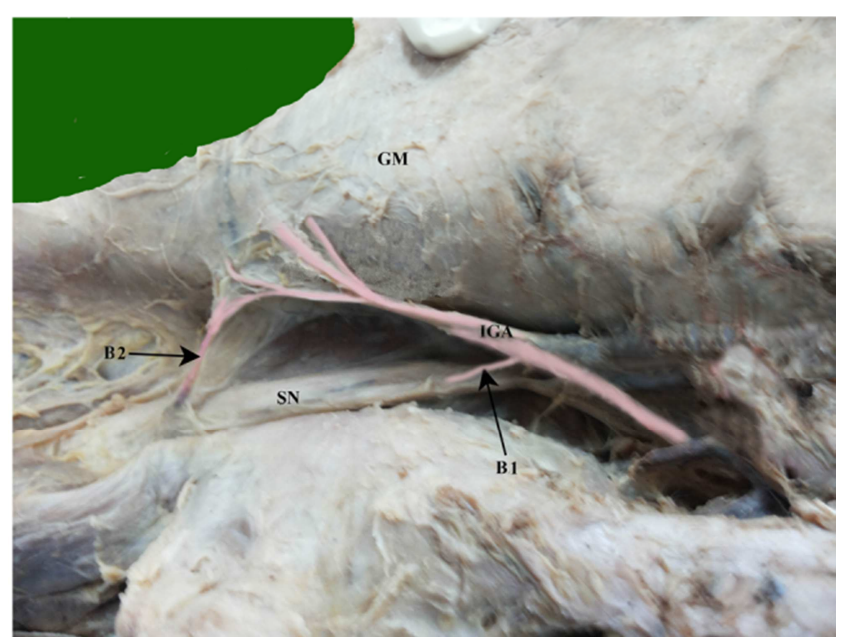

Fig. 3. A photograph of left lower limb with dissected gluteal region showing the inferior gluteal artery (IGA) giving 2 branches $(B 1, B 2)$ to supply the sciatic nerve (SN) from its posterior aspect. GM: gluteus maximus muscle. 


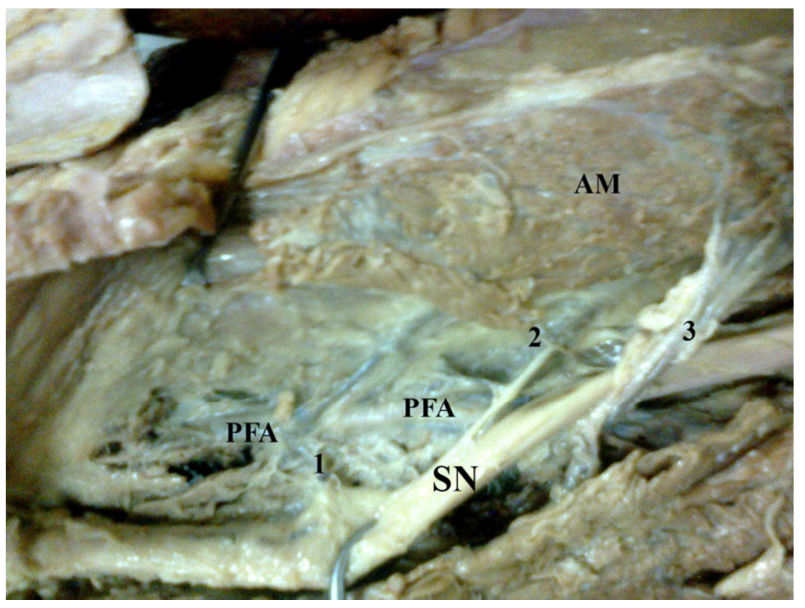

Fig. 4. A photograph of left lower limb with dissected posterior thigh showing profunda femoris artery (PFA) giving 3 perforators $(1,2,3)$ to supply the anteromedial aspect of sciatic nerve (SN). AM: adductor magnus muscle.

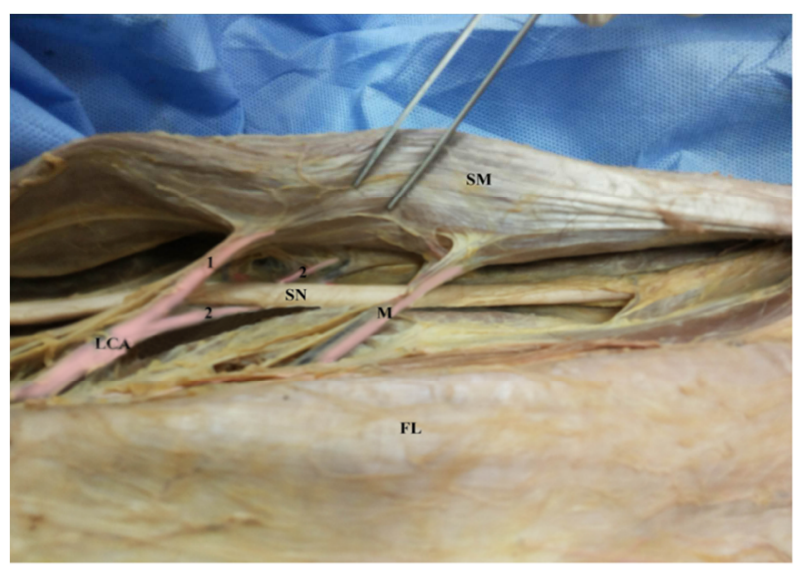

Fig. 5. A photograph of left lower limb with dissected posterior thigh showing the lateral circumflex artery (LCA) giving 2 branches $(1,2)$ to the posterior and anterior aspects of sciatic nerve (SN) respectively. M: muscular branch to semimembranosus muscle (SM). FL: fascia lata.

\section{Discussion}

Variations of the sciatic nerve anatomy and blood supply are complex and largely not dealt with in common anatomy texts. These variations should be well known to any surgeon operating in this anatomical region.

Nerves of the limbs, especially of the lower ones, are exposed to constant stretching and compression during everyday activities. However, in spite of that fact, nerve fibers normally conduct nerve impulses. Besides special connective tissue sheaths, which protect them from stretching and compression, the arterial arrangements of peripheral nerves may be designed to ensure maintenance of blood supply(3).

In the present study simultaneous ligation of profunda femoris and internal iliac arteries with revascularization of the limb through extraanatomic iliosuperficial femoral bypass using reversed saphenous vein graft resulted in paresis of the limb inspite of the felt distal pulsations(case 1). Also In chronic lower limb ischemic patient, with occluded profunda femoris artery and patent internal iliac artery. Accidental interruption of internal iliac artery blood flow resulted in sciatic ischemia (case2). While in case 3 occlusion of internal iliac artery with patent external iliac, common femoral and profunda femoris with superficial femoral artery (SFA), accidental interruption of profunda femoris artery (during construction of femoropopliteal bypass) resulted in lower limb paresis and sciatic ischemia by electrophysiological studies in presence of distal pulsations.

Teare et al (12), in their study found that, sciatic nerve ischemia occur after internal iliac artery occlusion by placement of balloon catheter. This differs from the present study that sciatic nerve ischemia occured only if there is simultaneous occlusion of profunda femoris and internal iliac arteries inspite of felt distal pulsations.

Eguchi and Majima(13) in their case report of thromboembolism secondary to profunda femoral artery aneurysm found that occlusion of profunda femoris artery by thrombus resulted in sciatic neuropathy after dissection of the aneurysm. According to the anatomical results of the present study, this may be due to concomitant occlusion of internal iliac artery which was not well investigatted in their study.

In the present study the sciatic nerve emerged from under cover of piriformis muscle and covered by gluteus maximus. There was high division of sciatic nerve into common peroneal and tibial component: in 6 out 20 specimens (30\%) it was in the gluteal region, in 6 out 20 specimens (30\%), it was in the upper third of thigh and in 8 out 20 specimens $(40 \%)$ it was in the middle of thigh. These findings are in agreement with Prathiba et al. (1) and Pokorny et al (2).

Andrew (14) stated that variations of the sciatic nerve anatomy can be divided into the height of division of its branches, relation of the branches to the piriformis muscle, and its blood supply. And stated that it is unknown whether these variations increase the risk of surgical injury and consequent morbidity.

In the present study, the sciatic artery originated from inferior division of IGA, supplied the posterior aspect of SN and continued with its tibial component. Also the IGA gives 2 branches to supply the posterior aspect of SN.

On the other hand, Ugrenovic et al (3) and Latarjet (4) found that the initial part of the sciatic nerve was accompanied and supplied by the sciatic artery (arteria comitans nervi ischiadici), a long slender vessel arising from or a continuation of the inferior gluteal artery which lies on the medial aspect of the nerve.

Williams et al (7) stated that, in the lower part of the thigh the sciatic artery which originated from inferior gluteal artery penetrated the sciatic nerve. A finding which is not in agreement with the present study in that inferior gluteal artery supplied the upper part of sciatic nerve because there was high division of it.

Schafer and Thane (8) found that a branch of the inferior gluteal artery (formerly called the sciatic artery), the "comes nervi ischiadici," anastomoses with twigs from the perforating arteries. A finding which is not in agreement with 
the present study in that the anastomosis was between the 1 st and 2 nd perforating arteries and internal pudendal artery.

In the present study, the $1 \mathrm{st}$ and 2 nd perforating arteries formed an anastomotic channel from which an artery ascends to supply the upper part of sciatic nerve. The third perforating artery ascends upward to supply the terminal part of SN and proximal part of its tibial component.

IPA supplied the anterior aspect of SN and anastomosed with the anastomosing branch of the first and second perforating arteries. This differs from the finding of Schafer and Thane (8) who found that a branch of the inferior gluteal artery anastomosed with twigs from the perforating arteries.

Karmanska et al. (9) observed that during its course in the gluteal region and thigh the sciatic nerve is supplied by between 4 and 8 nutrient arteries which arise from the inferior gluteal, medial circumflex femoral, perforating, and popliteal arteries. A finding which differs from the present study in that popliteal and medial circumflex artery doesn't share in the arterial supply of sciatic nerve due to higher division of sciatic nerve and lateral circumflex femoral artery replaced the medial circumflex femoral in its blood supply.

Also, Ugrenovic et al (3) in their study on thirty-six lower limbs to study the blood supply of the human sciatic nerves of fetuses. They found that the extraneural arterial chain of the sciatic nerve was composed of 2-6 arterial branches of the inferior gluteal artery, the medial circumflex femoral artery, the perforating arteries, and the popliteal artery.

In the present study the origin of arterial supply of sciatic nerve was variable. Six out of 20 specimens $(30 \%)$ it originated from the inferior gluteal artery, 6 out of 20 specimens $(30 \%)$ from first, second and third perforating arteries, 5 out of 20 specimens $(25 \%)$ from the lateral circumflex femoral artery and 3 out of 20 specimens (15\%) from internal pudendal artery.

Ugrenovic et al (3) in their study on arterial blood vessels of human fetal sciatic nerve found that the anastomotic arterial chain of the human sciatic nerve was observed in all cases. In $75 \%$ of the cases it was composed of the branches of the inferior gluteal artery, the medial circumflex femoral artery and the first two perforating arteries. The nutrient branch of the third perforating branch was less frequently (in $14.5 \%$ of the cases) part of this anastomotic arterial chain. This is in agreement with the present study except for the medial circumflex artery doesn't share in this anastomosis and also that the 3rd perforating artery had a higher percentage of share in this anastomosis.

On the other hand Georgakis and Soames (15) found that a total of 28 sciatic arteries were identified, of which $14(50 \%)$ arose from the medial circumflex femoral artery, 11(39\%) from the inferior gluteal artery, $2(7 \%)$ from the first perforating artery, and $1(4 \%)$ from the internal pudendal artery.

In the present study, anastomosis between internal iliac artery through its internal pudendal branch and external iliac artery through perforating arteries was observed.

Mikusek et al (16), in their study on vascularization of the human sciatic nerve fasciculi found that the arteries of fasciculi form intrafascicular vascular net.

In the present study, the mean length of the branches from the inferior gluteal artery was $34.9 \pm 1 \mathrm{~mm}$, third perforating artery was $26.7 \pm 0.2 \mathrm{~mm}$, from the anastomotic channel of the first and second perforating arteries was $22.6 \pm 1 \mathrm{~mm}$, from internal pudendal artery was $20 \pm 1 \mathrm{~mm}$ and from lateral circumflex femoral artery was $17 \pm 1 \mathrm{~mm}$.

Georgakis and Soames (15) stated that, except for sciatic arteries arising directly from the inferior gluteal artery, which had a mean length of $35.9 \mathrm{~mm}$, the mean length of the remaining arteries was between $15.8 \mathrm{~mm}$ (branches from the superior division of the inferior gluteal artery) and $22.7 \mathrm{~mm}$ (branches from the first perforating artery), these measurments are near to the measurments of the presnt study.

\section{Conclusion}

Over viewing our results we find that anastomosis between internal iliac artery through its internal pudendal branch and external iliac artery through perforating arteries was observed to be sufficient enough to prevent nerve ischemia during vascular surgery. Also the origin of the arteries supplying sciatic nerve is variable. In case of profunda femoris occlusion we should preserve internal pudendal artery and the reverse.

\section{References}

[1] Prathiba K, Seema M, Udaya KP and Dharmendar PN. A cadaveric study on anatomical variations of sciatic nerve in relation to pyriformis muscle in andhra pradesh region. IJCRR 2013; 5(21): 56-60.

[2] Pokorny D, Jahoda D, Veigl D, Pinskerova V and Sosna A. Topographic variations of the relationship of the sciatic nerve and the piriformis muscle and its relevance to palsy after total hip arthroplasty. Surg Radiol Anat 2006; 28:88-91.

[3] Ugrenovic SZ, Jovanovic ID, Kovacevic P, Petrovic S, and Simic T. Similarities and dissimilarities of the blood supplies of the human Sciatic, tibial, and common peroneal nerves. Clin. Anat. 2013; 26(7): 875-82.

[4] Latarjet A. 1929. Testut's Traite D'Anatomie Humaine. 8th Ed. Paris: Tome Deuxieme, Angeiologie, Systeme Nerveux, Central, Gaston, Doin and Cfe. p 349.

[5] Romanes GJ. 1981. Cunningham's Textbook of Anatomy. 12th Ed. Oxford: Oxford University Press. p 1376.

[6] Johnston TB, Whillis J. 1946. Gray's Anatomy. 29th Ed. London:Longmans Green. p 799.

[7] Standring S 2008. Gray's Anatomy. 40th Ed. Edinburgh: Churchill Livingstone. p 1562.

[8] Schafer EA, Thane GD. 1892. Quain's Anatomy, Vol. 2, Part II: Arthrology, Myology, Angeiology. London: Longmans Green. p 481.

[9] Karmanska W, Mikusek J, Karmanski A. Nutrient arteries of the human sciatic nerve. Folia Morphol (Warsz) 1993;52:209-15.

[10] Austin MS, Klein GR, Sharkey PF, Hozack WJ, Rothman RH. Late sciatic nerve palsy caused by hematoma after primary total hip arthroplasty. J Arthroplasty2004; 19:790-2. 
[11] Weil Y, Mattan Y, Goldman V, Liebergall M. Sciatic nerve palsy due to hematoma after thrombolysis therapy for acute pulmonary embolism after total hip arthroplasty. J Arthroplasty 2006; 21: 456-9.

[12] Teare J, Evans E, Belli A, Wendler R. Sciatic nerve ischaemia after iliac artery occlusion balloon catheter placement for placenta percreta. International Journal of Obstetric Anesthesia 2014: 23(2):178-81.

[13] Eguchi K and Majima M. Sciatic neuropathy caused by disorder of a nutrient artery: A case report of thromboembolism secondary to profunda femoral artery aneurysm. Archives of physical medicine and rehabilitation 2001;82 (2):253-5.
[14] Andrew JK. Variations of the sciatic nerve anatomy and blood supply in the gluteal region: a review of the literature. ANZ Journal of Surgery 2014; 84(11): 816-9.

[15] Georgakis E and Soames R. Arterial supply to the sciatic nerve in the gluteal region. Clin Anat. 2008; 21(1):62-5.

[16] Mikusek J1, Karamńska W and Karmański A. Vascularization of the human sciatic nerve fasciculi. Folia Morphol.1997;56(3):175-81. 